\title{
DESENVOLVIMENTO DE ROTINAS AUTOMATIZADAS PARA O DIMENSIONAMENTO DE PERFIS FORMADOS A FRIO SUBMETIDOS A SOLICITAÇÕES COMBINADAS
}

\author{
Felipe Castelli Sasso \\ Graduando em Engenharia Civil pela UPF, Passo Fundo, RS, Brasil. \\ castellisasso@gmail.com \\ Fernando Busato Ramires \\ Doutor em Engenharia Civil pela PUC-Rio, Rio de Janeiro, RJ, Brasil. \\ fernandoramires@upf.br
}

\section{RESUMO}

O presente artigo tem por objetivo o desenvolvimento de rotinas automatizadas para o dimensionamento de perfis de aço formados a frio visando o uso acadêmico. O mesmo justifica-se por auxiliar no desenvolvimento das atividades em disciplinas de estruturas de aço, fornecendo agilidade na resolução de exemplos. O desenvolvimento das rotinas baseou-se na norma brasileira ABNT NBR 14762:2012, da qual foi utilizado o Método da Seção Efetiva para o dimensionamento dos perfis. Para a automatização das mesmas, utilizou-se o software matemático SMath Studio Desktop. As rotinas mostraram-se eficientes e serão implementadas como material de apoio nas disciplinas de estruturas de aço na Universidade de Passo Fundo.

Palavras-chave: Perfil de aço formado a frio. Dimensionamento. Método da Seção Efetiva.

\section{DEVELOPMENT OF AUTOMATED ROUTINES FOR THE DESIGN OF COLD-FORMED SEC- TIONS SUBMITTED TO COMBINED REQUESTS}

\begin{abstract}
This article aims the development of automated routines for the design of cold-formed steel sections for academic use. The same, it is justified to help in the development of the academic activities of steel structures, providing agility in the resolution of examples. The implementation of the routines was based on the Brazilian Standard ABNT NBR 14762:2012, of which the Effective Section Method was used for the sections design. For the automation of them, the mathematical software SMath Studio Desktop was used. The routines are efficient and will be used as support material in the disciplines of steel structures at the University of Passo Fundo.
\end{abstract}

Keywords: Cold-formed steel section. Design. Effective Section Method. 


\section{INTRODUÇÃO}

As estruturas de aço permitem uma maior velocidade de execução e menores desperdícios de materiais quando comparadas as estruturas de concreto armado. Entre as estruturas de aço, hodiernamente, os perfis formados a frio (PFF) têm sido empregados em larga escala na construção civil, pois propiciam diversas vantagens como flexibilidade nas aplicações e baixo custo de fabricação se comparados aos perfis laminados e soldados. Além das vantagens, de acordo com Silva et al. (2014), os PFF são utilizados de forma eficiente em galpões de pequeno e médio porte, coberturas, mezaninos, casas populares e edifícios de pequeno porte.

Conforme Carvalho et al. (2014), em comparação aos perfis laminados, a produção de PFF é de baixo custo, pois, por ser composto de chapas delgadas, os mesmos possuem maior leveza, o que proporciona uma maior facilidade de fabricação, manuseio e transporte, não sendo necessários grandes maquinários para o seu içamento. Ainda, de acordo com Fadden (2006), a adoção desses perfis em estruturas de aço se mostra vantajosa não só pelo menor custo, mas também pela limitação da variedade de perfis laminados no Brasil.

$\mathrm{O}$ processo de dimensionamento dos $\mathrm{PFF}$, devido à grande quantidade de verificações de instabilidade, é extenso e trabalhoso. Nos cursos de graduação, usualmente, os acadêmicos utilizam planilhas digitais como auxílio na resolução de exemplos propostos. Porém, essas planilhas geralmente fornecem apenas os resultados finais para a verificação do exemplo, não contribuindo de forma significativa com o aprendizado do acadêmico. Desta forma, o presente trabalho tem por objetivo o desenvolvimento de rotinas de cálculo automatizadas para o dimensionamento de PFF, buscando fornecer auxílio digital de forma didática para a resolução de exemplos nas disciplinas de estruturas de aço.

Para o desenvolvimento das rotinas, foram utilizadas as normas brasileiras ABNT NBR 14762 - Dimensionamento de estruturas de aço constituídas por perfis formados a frio (2012) e ABNT NBR 6335 - Perfis estruturais de aço formados a frio - Padronização (2012). Ainda, para a automatização das rotinas, utilizou-se o software matemático SMath Studio Desktop (ANDREY IVASHOV, 2018).

As rotinas foram elaboradas para os perfis $U$ simples, $U$ enrijecido, $Z$ enrijecido 
à 90 e e $\mathrm{Z}$ enrijecido à $45^{\circ}$, os quais tiveram sua capacidade de trabalho calculada quando submetidos à tração, à compressão, à flexão simples e, foram verificados quanto ao momento fletor e força cortante combinados, à flexão oblíqua, à flexo-compressão e quanto ao limite de deslocamento.

\section{METOdOLOGIA}

A metodologia foi dividida em duas fases, sendo elas a elaboração de fluxogramas para as rotinas de cálculo e a implementação das rotinas no software SMath Studio Desktop, para a automatização das mesmas.

Um fluxograma genérico, ou seja, válido para todos os perfis analisados, foi desenvolvido para a organização da rotina para que uma vez inseridos os dados de entrada, todo o processo de dimensionamento do perfil, desde o cálculo das propriedades geométricas até a verificação do limite de deslocamento, fosse realizado de forma automática. A Figura 1 ilustra o fluxograma genérico desenvolvido. 
Figura 1 - Fluxograma genérico para a automatização das rotinas

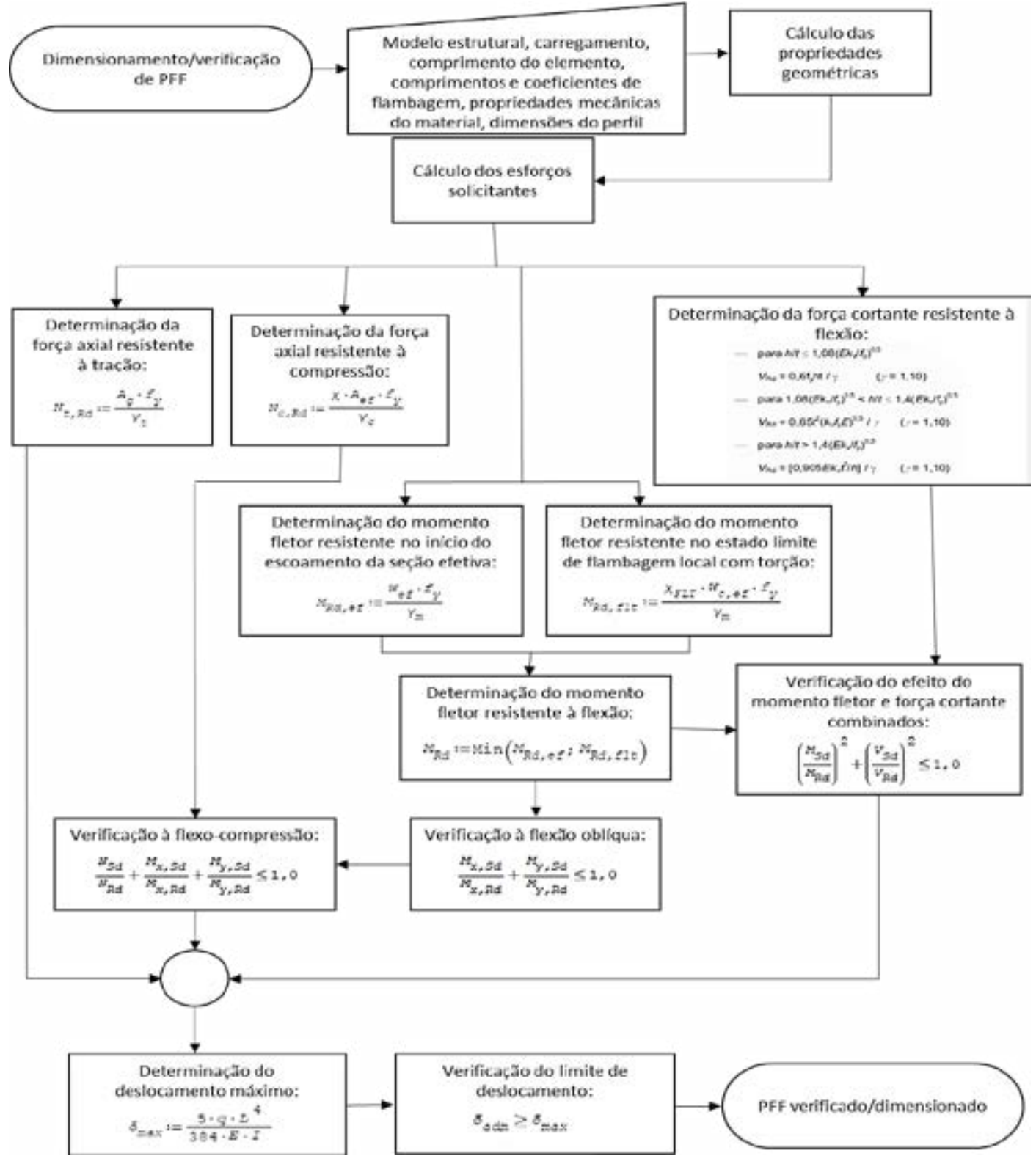

Fonte: (O autor, 2018)

Os dados de entrada a serem inseridos para o funcionamento das rotinas automatizadas são:

- Modelo estrutural;

- $\quad$ Carregamento;

- $\quad$ Comprimento do elemento; 
Figura 2 - Interface gráfica do software SMath Studio Desktop com uma rotina implementada

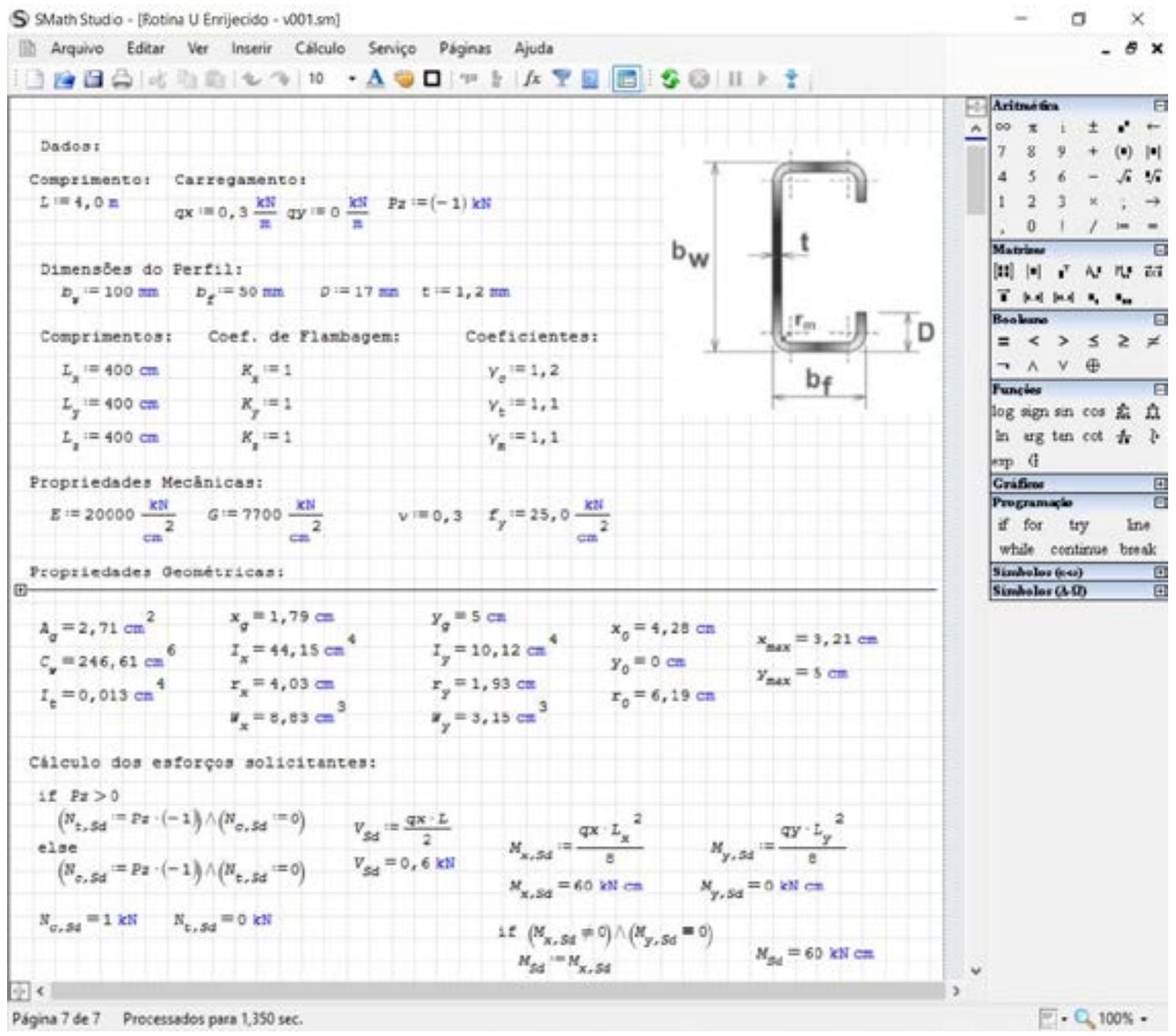

Fonte: (O autor, 2018)

\subsection{Propriedades geométricas}

As propriedades geométricas dos perfis foram determinadas de acordo com 0 Anexo A da norma ABNT NBR 6355:2012, a qual fornece expressões obtidas pelo emprego do Método Linear. Esse método consiste em admitir como concentrada toda a massa da seção do perfil na sua linha média e, os elementos são analisados como linhas retas e arcos (curvas). Os valores das propriedades dos elementos obtidos são multiplicados pela espessura da seção, de maneira a alcançar as propriedades geométricas desejadas. A Figura 3 ilustra os perfis para os quais foram desenvolvidas as rotinas e as dimensões utilizadas para a determinação das propriedades geométricas. 
Figura 3 - Dimensões utilizadas para o cálculo das propriedades geométricas
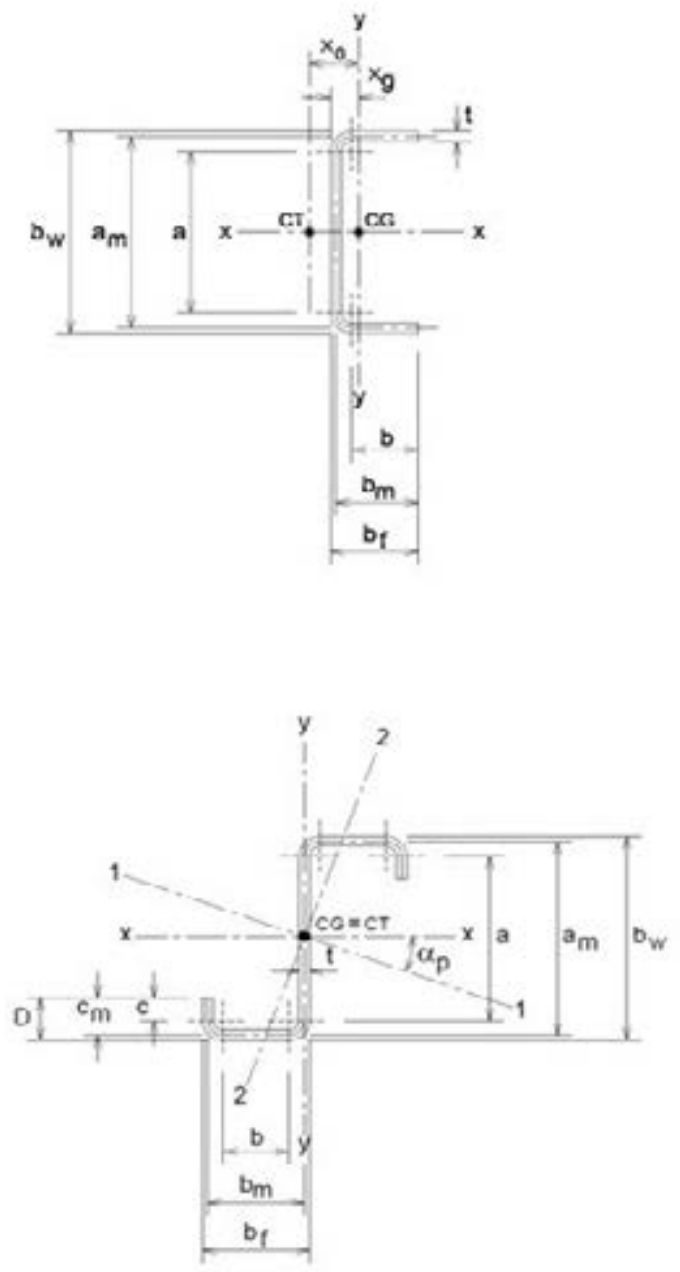
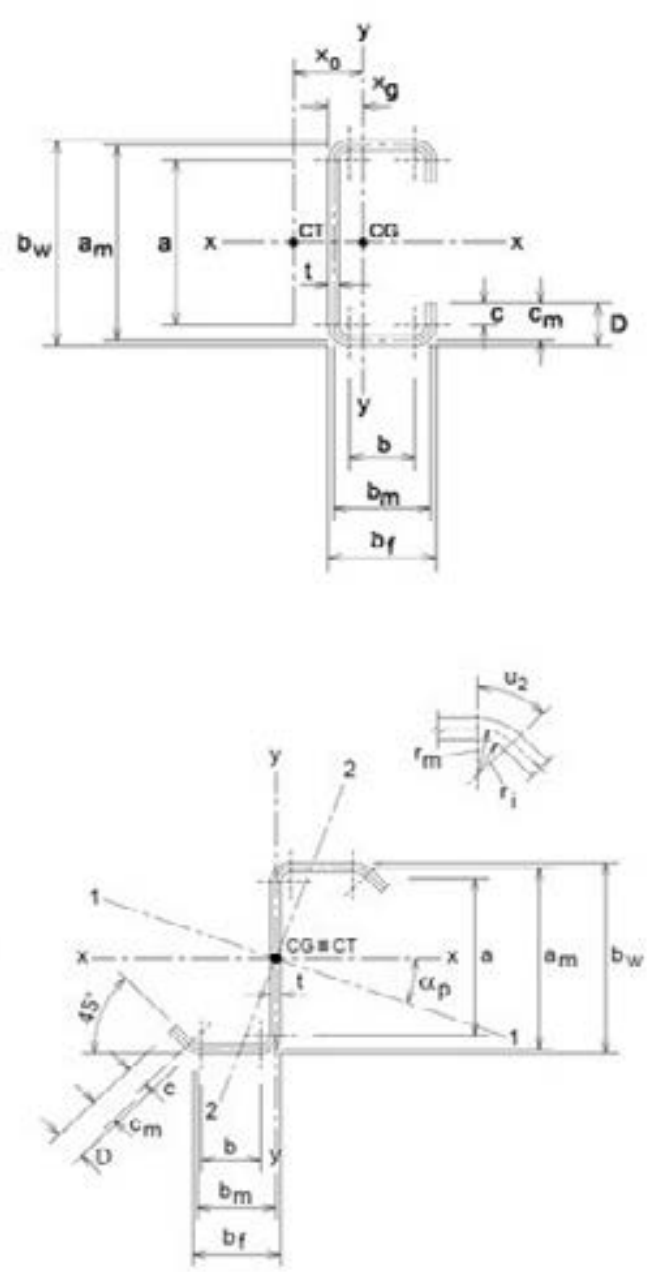

Fonte: (ABNT NBR 6355, 2012)

As expressões fornecidas pela ABNT NBR 6355:2012 para o cálculo das propriedades geométricas são relativamente extensas, o que dificulta a visualização dos usuários ao utilizar as rotinas automatizadas. Desta forma, todas as expressões foram inseridas em uma área que pode ser oculta, delimitada por uma ferramenta do software SMath Studio Desktop. Assim, são apresentados apenas os valores das propriedades já calculados para o usuário. Essa operação está ilustrada nas Figuras 4 e 5.

Figura 4 - Expressões fornecidas pela ABNT NBR 6355:2012 dentro da área a ser oculta 


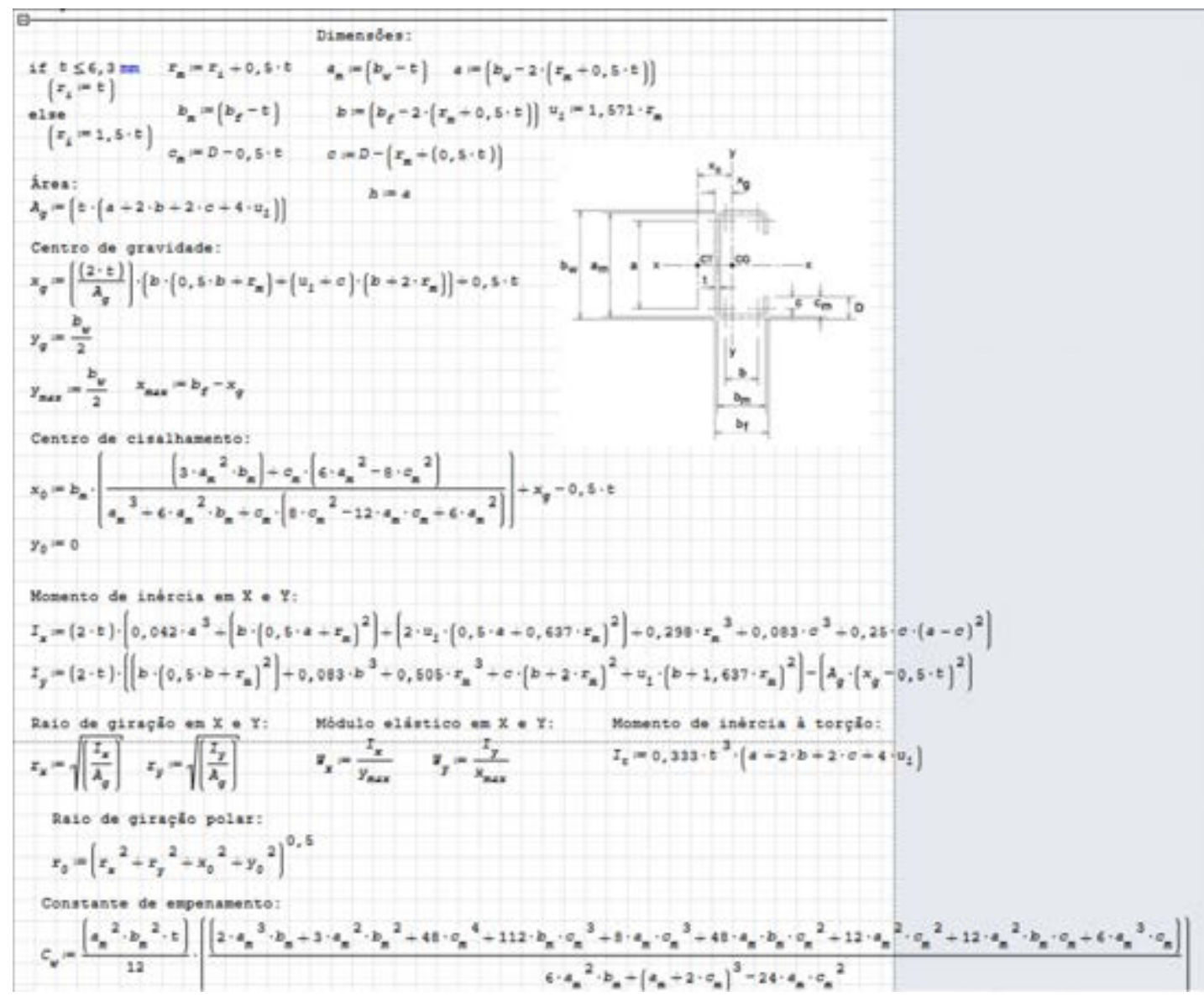

Fonte: (O autor, 2018)

Figura 5 - Visualização dos valores das propriedades geométricas

Propriedades Geometricas: ๑-

$\begin{array}{llllll}A_{g}=2,71 \mathrm{~cm}^{2} & x_{q}=1,79 \mathrm{~cm} & y_{\sigma}=5 \mathrm{~cm} & x_{0}=4,28 \mathrm{~cm} & x_{\max }=3,21 \mathrm{~cm} \\ C_{y}=246,61 \mathrm{~cm}^{6} & I_{x}=44,15 \mathrm{~cm}^{4} & I_{y}=10,12 \mathrm{~cm}^{4} & y_{0}=0 \mathrm{~cm} & y_{\max }=5 \mathrm{~cm} \\ I_{t}=0,013 \mathrm{~cm}^{4} & r_{x}=4,03 \mathrm{~cm} & r_{y}=1,93 \mathrm{~cm} & x_{0}=6,19 \mathrm{~cm} & \\ & x_{x}=8,93 \mathrm{~cm}^{3} & x_{y}=3,15 \mathrm{~cm}^{3} & & \end{array}$

Fonte: (O autor, 2018)

\subsection{Capacidade de trabalho}

A capacidade de trabalho dos perfis, ou seja, as forças axiais resistentes à tração e compressão, o momento fletor e a força cortante resistentes à flexão e, também as verificações quanto a esses efeitos combinados, foram determinados de acordo com a 
norma NBR 14762:2010. De acordo com Silva et al. (2014), o dimensionamento correto dos perfis formados a frio é dependente de uma análise não linear. Porém, podem ser empregadas expressões diretas, deduzidas a partir de teorias simplificadas e calibradas de forma empírica ou de teorias mais avançadas.

A norma brasileira ABNT NBR 14762:2012 fornece três métodos para a determinação da capacidade de trabalho dos perfis:

- Método das Larguras Efetivas (MLE) - é um processo iterativo e, segundo Silva et al. (2014), o cálculo das larguras efetivas dos elementos fornece as propriedades geométricas efetivas, ou seja, reduzidas da seção do perfil, as quais são consideradas para a verificação da instabilidade local;

- Método da Resistência Direta (MRD) - para sua utilização, deve-se ter conhecimento dos valores mínimos (críticos) das forças axiais e momentos fletores considerando os limites de comportamento elástico da seção de aço;

- Método da Seção Efetiva (MSE) - de acordo com Carvalho et al. (2014), consiste em expressões obtidas a partir de uma parametrização do MRD. É o método mais simples para a determinação dos esforços resistentes de forma manual.

Para o processo de dimensionamento nas rotinas, foi utilizado o MSE, pois é o mesmo método que os alunos utilizam para a resolução de exemplos nas disciplinas de estruturas de aço na Universidade de Passo Fundo.

\subsection{Barra submetida à tração}

Como as rotinas são para o dimensionamento considerando somente o elemento de barra, e não suas ligações, o efeito de tração não gera flambagem na mesma, dessa forma é necessária apenas a verificação ao escoamento da seção bruta, ou seja, a força resistente à tração ao longo da barra, a qual é calculada pela equação 1 . Salienta-se que não existe consideração da área efetiva para verificação da ruptura da seção líquida.

$$
\mathrm{N}_{\mathrm{t}, \mathrm{Rd}}=
$$

$\mathrm{N}_{\mathrm{t}, \mathrm{Rd}}$ - força axial de tração resistente de cálculo

- área bruta da seção transversal da barra

- resistência ao escoamento do aço

- coeficiente de ponderação (igual a 1,1) 


\subsection{Barra submetida à compressão}

Para o cálculo e verificação do esforço resistente da barra quando submetida à compressão, para a implementação da rotina no software, o processo de dimensionamento foi dividido em 3 passos:

- Verificação da esbeltez global - nessa etapa, determina-se a força axial de flambagem elástica que é utilizada para o cálculo do índice de esbeltez reduzido, o qual determina o fator de redução associado à compressão $(\chi)$;

- Determinação da área efetiva da seção do perfil pelo MSE - deve-se calcular o coeficiente de flambagem local por expressões fornecidas pela ABNT NBR 14762:2012, o qual é a utilizado para o cálculo da esbeltez reduzida que determina a área efetiva do perfil $\left(\mathrm{A}_{\mathrm{ef}}\right)$;

- Determinação da força axial resistente à compressão $\left(N_{c, R d}\right)$ - é obtida pela equação 2 , considerando $\gamma=1,2$.

$\mathrm{N}_{c, R d}=$

\subsection{Barra submetida à flexão simples}

Quanto ao efeito de flexão simples, são feitas as verificações do momento fletor e da força cortante resistentes à flexão e, também, da combinação dos dois efeitos.

O momento fletor resistente à flexão é o valor mínimo entre o momento fletor resistente no início do escoamento da seção efetiva e o momento fletor resistente no estado limite de flambagem local com torção.

Para a verificação da força cortante resistente à flexão, a norma ABNT NBR 14762:2012 fornece 3 equações para a sua determinação, que devem ser utilizadas de acordo com a relação entre a altura e a espessura do perfil.

A verificação quando os efeitos são combinados, é feita pela equação 3, a qual relaciona o momento fletor e a força cortante resistentes com seus esforços solicitantes.

$\mathrm{M}_{\mathrm{sd}}-$ momento fletor solicitante

$\mathrm{M}_{\mathrm{Rd}}$ - momento fletor resistente

$\mathrm{V}_{\mathrm{Sd}}$ - força cortante solicitante

$\mathrm{V}_{\mathrm{Rd}}$ - força cortante resistente 


\subsection{Demais verificações}

Além dos esforços resistentes calculados e verificados conforme mostrado nos itens anteriores, as rotinas automatizadas verificam a barra quanto à flexão oblíqua, à flexo-compressão e também ao deslocamento máximo.

As verificações quanto à flexão oblíqua e à flexo-compressão são realizadas pelas equações 4 e 5 , respectivamente, as quais relacionam a capacidade de trabalho do perfil e seus esforços solicitantes.

$M_{x, S d}-$ momento fletor solicitante em relação ao eixo $x$

$M_{x, R d}-$ momento fletor resistente em relação ao eixo $x$

$M_{y, s d}$ - momento fletor solicitante em relação ao eixo $y$

$\mathrm{M}_{\mathrm{y}, \mathrm{Rd}}$ - momento fletor resistente em relação ao eixo y

$\mathrm{N}_{c, S d}$ - força axial de compressão solicitante

$\mathrm{N}_{c, \mathrm{Rd}}$ - força axial resistente à compressão

Já na verificação do deslocamento máximo, é realizada uma comparação entre o deslocamento máximo atuante e o limite de deslocamento, de acordo com a situação analisada, determinado pelo Anexo A da norma ABNT NBR 14762:2012.

\section{RESULTADOS E DISCUSSÃO}

Para a validação das rotinas automatizadas, as mesmas foram aplicadas aos exemplos contidos na obra de Silva et al. (2014). As comparações para efeito de validação das rotinas podem ser vistas nas Tabelas 1 e 2.

Tabela 1 - Validação das rotinas automatizadas para barra submetida à compressão

\begin{tabular}{|c|c|c|c|c|c|c|c|c|}
\hline \multicolumn{9}{|c|}{ BARRA SUBMETIDA À COMPRESSÃO } \\
\hline & $\mathrm{N}_{\mathrm{ex}}(\mathrm{kN})$ & $\mathrm{N}_{\mathrm{ey}}(\mathrm{kN})$ & $\begin{array}{c}N_{\mathrm{ez}} \\
(\mathrm{kN})\end{array}$ & $\mathrm{N}_{\mathrm{exz}}(\mathrm{kN})$ & $\mathrm{N}_{\mathrm{e}}(\mathrm{kN})$ & $x$ & $A_{e f}\left(\mathrm{~cm}^{2}\right)$ & $\begin{array}{l}N_{c, R d} \\
(k N)\end{array}$ \\
\hline $\begin{array}{c}\text { Exemplo } \\
04 \\
\text { (SILVA et } \\
\text { al., 2014) }\end{array}$ & 54,47 & 12,49 & 10,55 & 9,57 & 9,57 & 0,124 & 2,71 & 7,0 \\
\hline
\end{tabular}




\begin{tabular}{|c|c|c|c|c|c|c|c|c|}
\hline $\begin{array}{c}\text { Rotina } \\
\text { automati- } \\
\text { zada }\end{array}$ & 54,47 & 12,48 & 10,55 & 9,58 & 9,58 & 0,124 & 2,71 & 7,0 \\
\hline
\end{tabular}

Fonte: (O autor, 2018)

Tabela 2 - Validação das rotinas automatizadas para barra submetida à flexão simples

\begin{tabular}{|c|c|c|c|c|c|c|c|c|c|}
\hline \multicolumn{7}{|c|}{ BARRA SUBMETIDA À FLEX ̃̃O SIMPLES } \\
\hline $\begin{array}{c}\mathrm{W}_{\text {ef }} \\
\left(\mathrm{cm}^{3}\right)\end{array}$ & $\begin{array}{c}\mathrm{M}_{\mathrm{Rd}, \mathrm{ef}} \\
(\mathrm{kN} . \mathrm{cm})\end{array}$ & $\begin{array}{c}\mathrm{M}_{\mathrm{e}} \\
(\mathrm{kN} . \\
\mathrm{cm})\end{array}$ & $\lambda_{0}$ & $\chi_{\mathrm{FLT}}$ & $\begin{array}{c}\mathrm{W}_{\text {ef }} \\
\left(\mathrm{cm}^{3}\right)\end{array}$ & $\begin{array}{c}\mathrm{M}_{\mathrm{Rd}, \mathrm{ft}} \\
(\mathrm{kN} . \mathrm{cm})\end{array}$ & $\begin{array}{c}\mathrm{M}_{\mathrm{Rd}}(\mathrm{kN} . \\
\mathrm{cm})\end{array}$ & $\mathrm{V}_{\mathrm{Rd}}(\mathrm{kN})$ \\
\hline $\begin{array}{c}\text { Exem- } \\
\text { plo 07 } \\
\begin{array}{c}\text { e 09 } \\
\text { (SILVA } \\
\text { et al., } \\
\text { 2014) }\end{array}\end{array}$ & 8,6 & 195,45 & 71,1 & 1,762 & 0,322 & 8,83 & 64,6 & 64,6 & 13,45 \\
\hline $\begin{array}{c}\text { Rotina } \\
\text { auto- } \\
\text { mati- } \\
\text { zada }\end{array}$ & 8,6 & 195,35 & 71,05 & 1,763 & 0,322 & 8,83 & 64,6 & 64,6 & 13,45 \\
\hline
\end{tabular}

Fonte: (O autor, 2018)

Os exemplos da obra são resolvidos com a aplicação do Método das Larguras Efetivas e, como pode ser visto nas tabelas, os valores resultantes são muito próximos aos resolvidos pelas rotinas automatizadas, nas quais é utilizado o Método da Seção Efetiva. Porém, essa proximidade de valores pode não ocorrer, pois ao analisar os exemplos resolvidos na obra de Carvalho et al. (2014), onde os autores resolvem os mesmos exemplos utilizando ambos os métodos (MLE e MSE), pode-se perceber uma diferença de até $15 \%$ nos valores resultantes. 


\section{CONSIDERAÇÕES FINAIS}

Pode-se concluir que as rotinas automatizadas se mostraram eficientes e fornecem agilidade na verificação da capacidade de trabalho dos perfis formados a frio, pois uma vez inseridos os dados de entrada, elas calculam todos os parâmetros automaticamente. Além disso, por fornecerem uma visualização do processo de dimensionamento de forma didática, podem auxiliar discentes e docentes na resolução de exemplos de dimensionamento de PFF. Desta forma, as mesmas serão implementadas como material de apoio nas disciplinas de estruturas de aço na Universidade de Passo Fundo. Ainda, de forma a complementar o trabalho realizado, estuda-se a possibilidade de desenvolvimento de um aplicativo para smartphone que utilize o processo de dimensionamento automatizado implementado nas rotinas, de forma a aliar cada vez mais o ensino à tecnologia.

\section{REFERÊNCIAS}

ANDREY IVASHOV. SMath Studio Desktop. 2018. Disponível em: https://en.smath.com/ view/SMathStudio/summary. Acesso em: 21 dez. 2018.

ASSOCIAÇÃO BRASILEIRA DE NORMAS TÉCNICAS. NBR 6355: Perfis estruturais de aço formados a frio - Padronização. Rio de Janeiro, 2012.

. NBR 14762: Dimensionamento de estruturas de aço constituídas por perfis formados a frio. Rio de Janeiro, 2012.

CARVALHO, P. R. M. de; GRIGOLETTI, G.; BARBOSA, G. D. Curso básico de perfis de aço formados a frio. 3. ed. Porto Alegre: [s. n.], 2014.

FADDEN, Wesley Marton Mac. Estudo comparativo de perfis de aço laminados e formados a frio utilizados em terças de cobertura. Trabalho de Conclusão de Curso (Graduação em Engenharia Civil), Universidade Estadual de Londrina. Londrina, 2006. 
SILVA, E. L.; PIERIN, I.; SILVA, V. P. e. Estruturas compostas por perfis formados a frio: dimensionamento pelo método das larguras efetivas e aplicação conforme ABNT NBR 14762:2010 e ABNT NBR 6355:2012. Rio de Janeiro: Instituto Aço Brasil / CBCA, 2014. 